\title{
Avaliação de ferramenta informatiza- da para gestão de resíduos em um hospital universitário de nível terciário
}

\section{Evaluation of computerized tool for waste management in a tertiary level teaching hospital}

André L. Vitor' ${ }^{1}$, David Falango', Heloisa B. D. Oliveira1', Jorge R. Lopes Junior ${ }^{1}$, Lucas R. Pellozo', Luzimar R. M. F. Borges ${ }^{1}$, Regina C. N. Pontelli', Maria Eulália L. V. Dallora ${ }^{2}$

\begin{abstract}
RESUMO
Modelo de Estudo: Estudo Original

Introdução: Os avanços tecnológicos nas ultimas décadas na área de informática são incontestáveis. Sistemas informatizados colaboram com a gestão, permitindo monitoramento e controle, auxiliando na tomada de decisão. A sugestão de um sistema informatizado para auxiliar os gestores da área de Gerenciamento Ambiental do Hospital das Clínicas da Faculdade de Medicina da Universidade de São Paulo (HCFMRP USP) tem a intenção de oferecer agilidade e praticidade na gestão de resíduos, principalmente os resíduos de saúde, proporcionando um acompanhamento de toda a cadeia, da segregação ao destino final. Objetivo: Apresentar e avaliar recursos de gestão de um sistema Informatizado para cadastro, controle e gerenciamento das informações sobre os resíduos. Métodos: Dados para simulação e avaliação da ferramenta foram obtidos na enfermaria da hematologia do HCFMRP. Após, análises qualitativas de pontos fortes e fracos foram realizadas juntamente com entrevistas feitas com os responsáveis pelo Serviço de Gerenciamento Ambiental do hospital. Resultados: A simulação permitiu uma análise completa das funcionalidades do sistema, juntamente com o fluxo de informações que deve ser aplicado para seu funcionamento. Conclusão: A ferramenta sugerida vai de encontro às necessidades do Serviço, o que otimiza a execução das tarefas e agiliza os processos. O Sistema informatizado permite a gestão dos resíduos desde a identificação dos usuários e seus respectivos resíduos produzidos, até seu destino final, incluindo a etapa de coleta.
\end{abstract}

Palavras Chave: Gestão ambiental; Gerenciamento de Resíduos; Sistema informatizado para Gestão de Resíduos; Resíduos de Serviços de Saúde.

1. Mestrandos em Organização de Saúde pelo Hospital das Clínicas - Faculdade de Medicina de Ribeirão Preto (HCFMRPUSP).

2. Diretora da Assessoria Técnica do HCFMRP-USP. Docente do Mestrado Profissional Gestão de Organizações de Saúde da Faculdade de Medicina de Ribeirão Preto - USP
Maria Eulália Lessa do Valle Dallora Hospital das Clínicas da Faculdade de Medicina de Ribeirão

Preto - Avenida Bandeirantes, 3900 CEP: 14048-900 - Ribeirão Preto (SP), Brasil meulalia@ hcrp.usp.br 


\begin{abstract}
Study model: Original study

Introduction: Technological advances over the last few decades in the it area are undeniable. Computerized systems collaborate with management, enabling monitoring and control, assisting in decision-making. The suggestion of a computerized system to assist managers in the area of Environmental Management of the Clinical Hospital Faculty of medicine the University of São Paulo (USP HOSPITAL) intends to offer agility and practicality in waste management, especially the waste of health, providing a complete monitoring of the entire chain, from segregation to the final destination. Objective: to present and evaluate resources for the administration of a computerized system to record, control, and management of information on waste. Methods: data for simulation and evaluation of the tool were obtained in the infirmary HOSPITAL hematology. After that, qualitative analyses of strengths and weaknesses were held along with interviews with those responsible for the environmental management of the hospital service. Results: the simulation allowed a complete analysis of the functionality of the system, along with the flow of information that should be applied for its functioning. Conclusion: the suggested tool meets the needs of the service, which optimizes the performance of the tasks and streamlines processes. The computerized system allows the management of waste from the identification of users and their respective waste produced, until its final destination, including the step of collecting.
\end{abstract}

Keywords: Environmental Management; Waste Manegement; Computerized System for Waste Management; Medical Waste.

\section{Introdução}

Os avanços tecnológicos nas ultimas décadas na área de informática são incontestáveis. Sistemas informatizados colaboram com a gestão, permitindo monitoramento e controle, auxiliando na tomada de decisão. A sugestão de um sistema informatizado para auxiliar os gestores da área de Gerenciamento Ambiental do Hospital das Clínicas da Faculdade de Medicina da Universidade de São Paulo (HCFMRP USP) tem a intenção de oferecer agilidade e praticidade na gestão de resíduos , principalmente os resíduos de saúde, proporcionando acompanhamento de toda a cadeia, da segregação ao destino final.

Em se tratando de assuntos ambientais, a utilização de sistemas informatizados torna-se uma ferramenta importante para as empresas buscarem uma administração eficaz e eficiente. Os assuntos relacionados a preservação e cuidados com o meio ambiente e com os seres vivos que o habitam, começam a ter um papel significativo na maneira de reformular os conceitos empresariais, quer por um olhar mais consciente, quer por exigências legais. Para a maioria das empresas, existem grandes desafios em se tratando de desenvolvimento sustentável, exigindo diversas ações e iniciativas nessa nova configuração empresarial. ${ }^{1}$

Um dos desafios é a manutenção da qualidade dos serviços, com o menor custo econômico, ambi- ental e social. Nesse contexto, as proposições das normas ISO 14000 possibilitam um correto posicionamento das empresas com relação à sociedade, através do respeito ao meio ambiente e da qualidade e competitividade de seus produtos e serviços. ${ }^{2,3}$

Entende-se que os processos para melhorias de desempenho ambiental e implantação de sistemas de gestão ambiental são precedidos por uma fase de diagnóstico, identificação dos aspectos e impactos ambientais e a preparação de um Plano de Ação, o qual deve ser baseado num relatório de Auditoria Interna Ambiental, com consequente formulação de uma política ambiental. ${ }^{4}$

Torna-se necessário o entendimento de que o investimento na questão ambiental não é uma medida para aumentar custos, e sim para reduzi-los, com a possibilidade do aumento na efetividade de diversas etapas do processo produtivo. ${ }^{5}$

Com o aumento das pressões legais os serviços de saúde, necessitam se adequar para contribuir na manutenção e cuidados com o meio ambiente. Nesse contexto, os hospitais constituem-se em grandes produtores de resíduos, dentre eles infectantes e químicos, que necessitam de uma gestão eficiente.

Os Resíduos de Serviços de Saúde (RSS) constituem-se em um problema de difícil solução, especialmente em instituições de grande porte e altamente especializadas. 
De acordo com a RDC n 306/04, e a Resolução $n^{\circ} 358 / 05$, são definidos como geradores de RSS todos os serviços relacionados com o atendimento à saúde humana ou animal, inclusive os serviços de assistência domiciliar e de trabalhos de campo Esses resíduos são classificados em cinco grupos, conforme suas características. ${ }^{6,7}$

De acordo com a Associação Brasileira de Normas Técnicas (ABNT), através da Norma Técnica NBR 10. 004:2004, os tipos de resíduos sólidos apresentam outra classificação, quanto aos seus riscos potenciais ao meio ambiente e à saúde pública, são classificados em quatro classes. ${ }^{8}$

A gestão de resíduos visa o gerenciamento em todas as fases do manejo: segregação, condicionamento, armazenamento, coleta, transporte, tratamento e disposição final ${ }^{9}$.

Os custos do tratamento dos resíduos tornamse cada vez mais elevados, sendo essencial a redução na geração de resíduos. Ferramentas de avaliação dessas atividades podem auxiliar no monitoramento das práticas implantadas e seu entendimento pode levara racionalização de recursos. ${ }^{10}$

A segregação de resíduos realizada de forma apropriada na unidade geradora pode reduzir a quantidade de resíduos perigosos, o custo de seu tratamento e o risco de contaminação ambiental.

As mudanças oriundas da Política Nacional de Resíduos Sólidos (Lei Federal no 12. 305/10) e seus desdobramentos tornam ainda mais relevante à existência de um sistema de "Gerenciamento de Resíduos de Serviços de Saúde" (GRSS) que busque melhoria contínua desse processo gerencial e que possa facilitar a atualização dos participantes. ${ }^{11}$.

Com bases nesses dispositivos legais citados e de acordo com as características dos resíduos gerados, o Plano de GRSS foi elaborado e implementado no Complexo do Hospital das Clínicas da Faculdade de Medicina da Universidade de São Paulo (HCFMRP USP).

O gerenciamento dos resíduos sólidos de saúde (RSS) gerados dentro do complexo HCFMRP está alicerçado em dispositivos legais que fornecem as diretrizes para a conduta correta, desde a segregação até o descarte final.

O Setor de Gerenciamento Ambiental iniciou as atividades no ano de 2002, vinculado ao Centro Integrado da Qualidade. Suas ações ambientais efetivas tiveram início no ano de 2003, com o mapeamento de todas as unidades (administrativas, assistenciais, operacionais e externas). Houve também a identificação, em cada setor, de qual o resíduo gerado e a sua quantidade produzida diariamente. Este setor consta com uma estrutura organizacional própria e comple$\mathrm{xa}$, conforme descrito na figura 1 .

A gestão dos resíduos foi uma das primeiras áreas a serem abordadas. O fato de tratar-se de um Hospital de grande porte e de nível terciário de atenção à saúde (com aproximadamente de 870 leitos) resulta na geração de grande volume de resíduos.

Com a comercialização dos recicláveis e venda de filme de raio $\mathrm{x}$, foi possível obtenção de recursos financeiros que resultou na ampliação da área física da Central de Resíduos, permitindo melhor acondicionamento dos resíduos hospitalares, e premiações por ações inovadoras tais como: Amigo do Meio Ambiente e Hospitais Saudáveis.

A Tabela 1 apresenta informações quanto à quantidade de resíduos coletados de 2007 a 2013, de acordo com os respectivos grupos.

Atualmente, o processo de solicitação de recolhimento dos resíduos químicos gerados pelas unidades é realizado conforme apresentado na figura 2.

A atual rotina do Serviço de Gerenciamento Ambiental não permite identificar a quantidade e o tipo de resíduos gerados pelas áreas, dificultando ações imediatas quando necessárias. Desta forma levanta-se a questão do que pode ser realizado para a melhoria da gestão dos resíduos químicos gerados no HCFMRP. Uma opção é a utilização de um sistema informatizado com potencial para aperfeiçoar a Gestão de Resíduos, e assim, melhorar as informações, rastreabilidade e orientações.

\section{Objetivo}

Apresentar e avaliar recursos de gestão de um sistema Informatizado online para cadastro, controle e gerenciamento das informações sobre os resíduos, desde a geração até a destinação final.

\section{Metodologia}

A metodologia para avaliar a ferramenta, desenvolvida para apoio à gestão de resíduos (GRSS), esta dividida da seguinte maneira:

- Seleção e apresentação da ferramenta utilizada;

- Seleção dos dados para simulação da ferramenta;

- Aplicação das análises escolhidas para avaliação da ferramenta; 


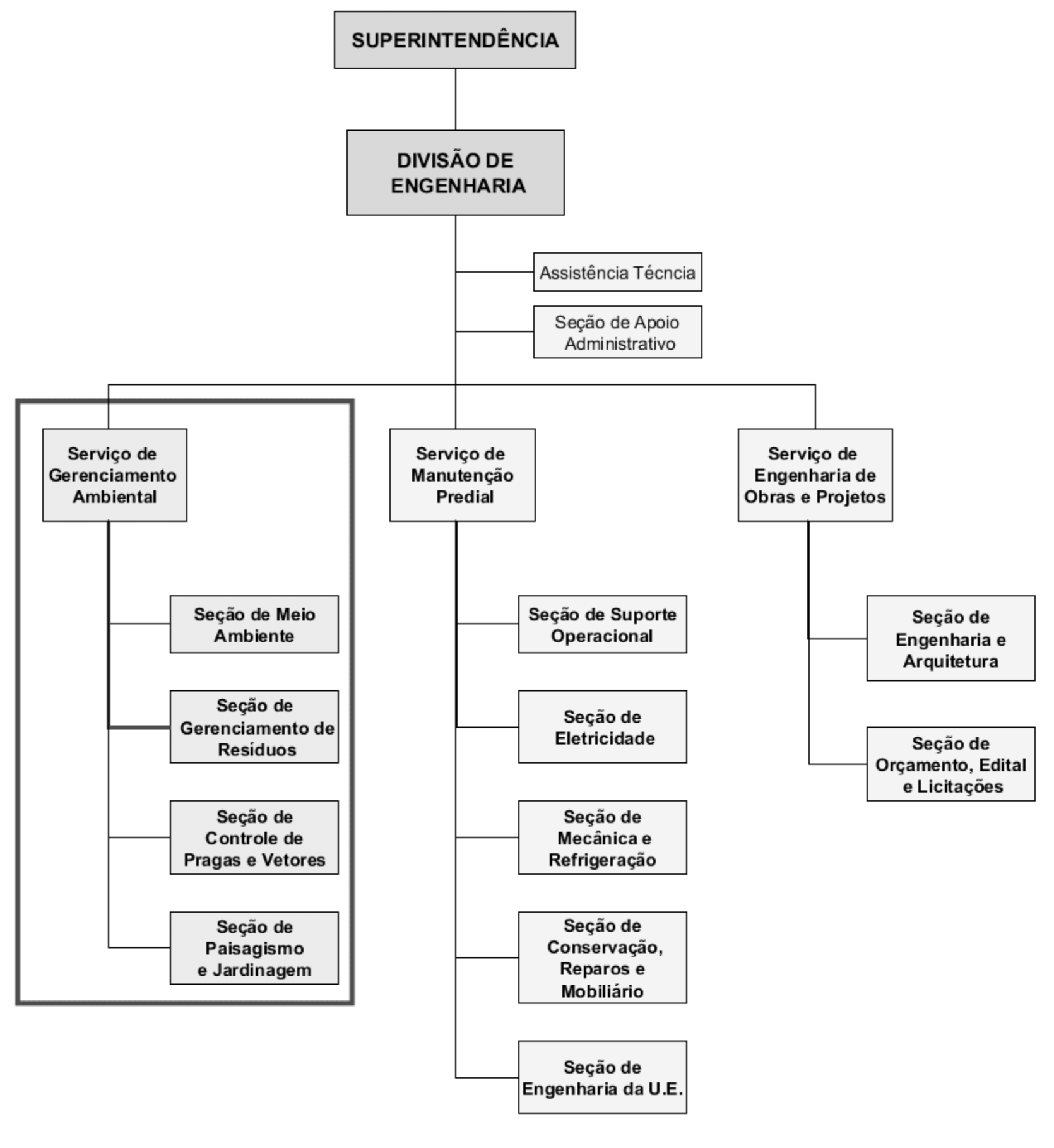

Figura I: Organograma do Serviço de Gerenciamento Ambiental do HCFMRP-USP. 
Tabela1.Resíduos Gerados no HCFMRP (por quilogramas).

\begin{tabular}{lccccc}
\hline Ano & $\begin{array}{c}\text { Resíduos Infectantes e } \\
\text { Perfuro-cortantes } \\
\text { (Grupos A e E) }\end{array}$ & $\begin{array}{c}\text { Resíduos } \\
\text { Químicos } \\
\text { (Grupo B) }\end{array}$ & $\begin{array}{c}\text { Rejeitos } \\
\text { Radioativos } \\
\text { (Grupo C) }\end{array}$ & $\begin{array}{c}\text { Resíduos Comuns } \\
\text { Refugo } \\
\text { (Grupo D) }\end{array}$ & $\begin{array}{c}\text { Resíduos Comuns } \\
\text { Recicláveis } \\
\text { (Grupo D) }\end{array}$ \\
\hline 2007 & $774.000 \mathrm{~kg}$ & $4.040 \mathrm{~kg}$ & $106 \mathrm{~kg} \mathrm{e} \mathrm{471}$ & - & $132.279 \mathrm{~kg}$ \\
2008 & $622.800 \mathrm{~kg}$ & $4.655 \mathrm{~kg}$ & $20 \mathrm{~kg}$ & - & $166.302 \mathrm{~kg}$ \\
2009 & $701.280 \mathrm{~kg}$ & $7.965 \mathrm{~kg}$ & $293 \mathrm{~kg}$ & - & $196.397 \mathrm{~kg}$ \\
2010 & $712.080 \mathrm{~kg}$ & $6.324 \mathrm{~kg}$ & $196 \mathrm{~kg}$ & - & $201.575 \mathrm{~kg}$ \\
2011 & $576.720 \mathrm{~kg}$ & $5.237 \mathrm{~kg}$ & $189 \mathrm{~kg}$ & $578.916 \mathrm{~kg}$ & $211.031 \mathrm{~kg}$ \\
2012 & $666.504 \mathrm{~kg}$ & $19.357 \mathrm{~kg}$ & $193 \mathrm{~kg}$ & $912.500 \mathrm{~kg}$ & $220.068 \mathrm{~kg}$ \\
2013 & $651.554 \mathrm{~kg}$ & $11.384 \mathrm{~kg}$ & $300 \mathrm{Kg}$ & $714.023 \mathrm{~kg}$ & $214.610 \mathrm{~kg}$ \\
\hline
\end{tabular}

Fonte: Relatório de Atividades do HCFMRP, 2013.

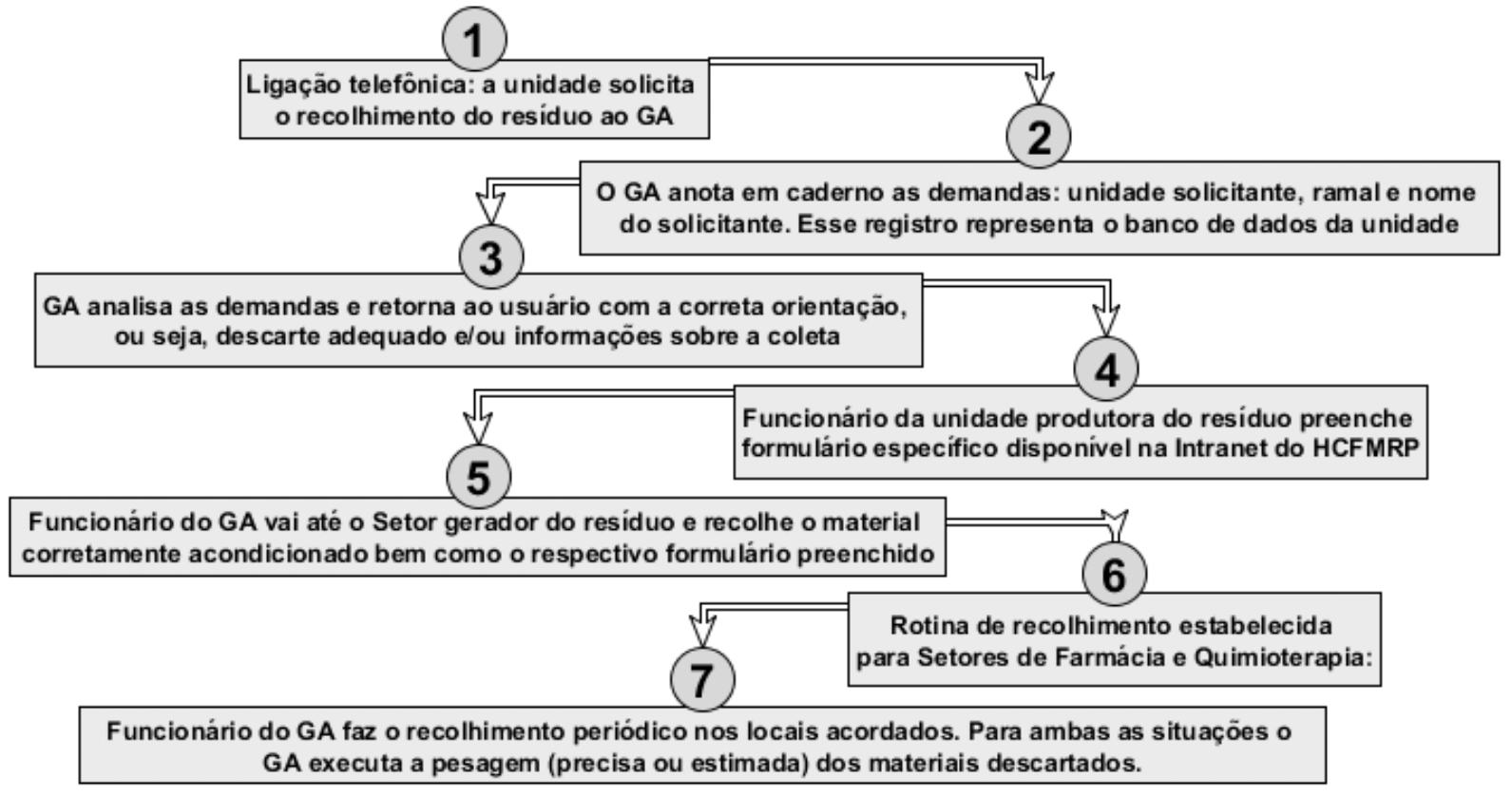

Figura 2: Processo atual de solicitação de recolhimento de resíduos.

\section{Ferramenta selecionada}

A ferramenta selecionada denomina g2r (Gestão de Resíduos em Rede) é um sistema desenvolvido e mantido pela empresa Bioprax - Soluções para Meio Ambiente, com sede na cidade de Ribeirão Preto/SP.

A Bioprax é uma empresa que esteve incubada na SUPERA, Incubadora de empresas de base tecnológica de Ribeirão Preto, na unidade vinculada e localizada no Hemocentro pertencente ao complexo HCFMRP-USP. A proximidade da Incubadora com o
HCFMRP permitiu o contato com a empresa, possibilitando sua liberação para utilização na presente pesquisa, com acesso a ferramenta e simulação com dados do Hospital.

\section{Dados simulados na ferramenta}

Para as simulações na ferramenta foram utilizados os dados referentes ao período de Dezembro de 2013 até Fevereiro de 2014, para o resíduo do Grupo B (Classificação RDC 306), RPM - Resíduos Perigosos de Medicamentos, produzidos pela enferma- 
ria de Hematologia, conforme consta no Gráfico 01. Estes dados foram disponibilizados pela equipe de Gerenciamento Ambiental.

\section{Análises selecionadas para avaliação da ferramenta}

As análises qualitativas utilizadas para avaliação da ferramenta foram:

- Análise crítica de pontos fortes e fracos sob a ótica do grupo de pesquisadores.

- Análise qualitativa realizada através das avaliações recebidas após apresentação do sistema para o Serviço de Gerenciamento Ambiental.

\section{Resultados}

A simulação permitiu uma análise das funcionalidades do sistema, juntamente com o fluxo de informações que deve ser aplicado para seu funcionamento.

\section{Apresentação da ferramenta}

O sistema web g2r é uma ferramenta que concentra informações relacionadas ao processo de gestão dos resíduos. Esta solução é baseada essencialmente em um sistema computacional voltados para a busca e proposição de alternativas de destinação dos resíduos às empresas.
O objetivo principal da ferramenta é auxiliar os envolvidos em diferentes processos e tarefas relevantes para o desenvolvimento de suas atividades de trabalho dentro da GR, centralizando as informações, simplificando processos relativos e potencializando relacionamentos. Dentre as funcionalidades disponibilizadas pela ferramenta estão:

- O cadastro de cada resíduo produzido, bem como a sua classificação perante as normas da ABNT 10004, CONAMA 313, ANTT e RDC 306; ${ }^{12,13}$

- Cadastro dos setores, bem como quais resíduos são produzidos em cada setor;

- Para cada resíduo são cadastradas alternativas de gestão e destinação: Disposição, Tratamento ou Reutilização.

- Para cada Alternativa de gestão pode se cadastrar as entidades envolvidas por do tipo: receptora, transportadora, coletora, etc. E armazenar arquivos relacionados a aquela alternativa, como, laudos, fotos da coleta, etc. ;

- O cadastro do fluxo de resíduos produzidos, formado pela informação da data, quantidade produzida, setor e tipo de armazenamento;

- O cadastro do fluxo de saídas/destinação do resíduos, formado pela informação da data, quantidade, valor de receita agregada ou custos de destinação e para qual daquelas alternativas de gestão cadastradas previamente foi destinado;

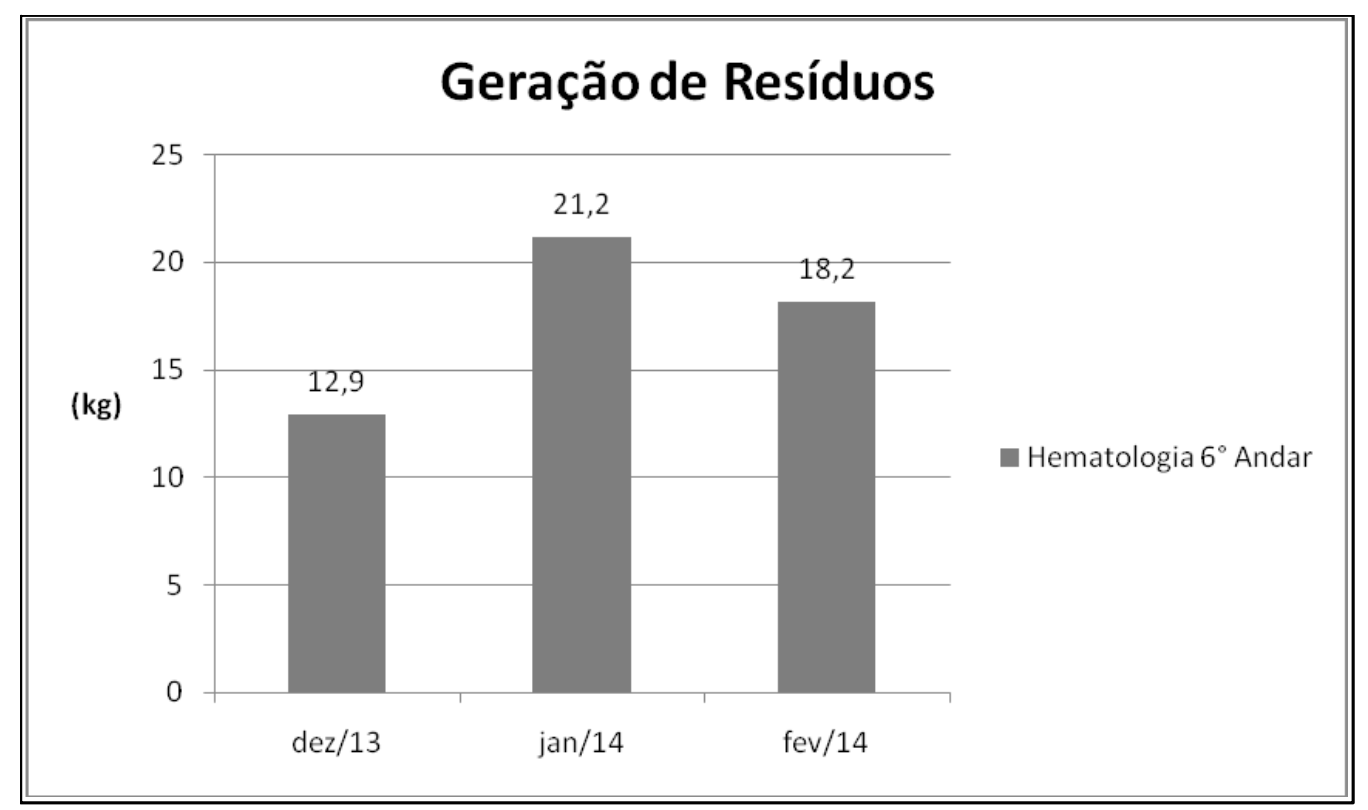

Gráfico 1. Produção de resíduos químicos da enfermaria de Hematologia do HCFMRP, no período de dezembro de 2013 a fevereiro de 2014. 
- Relatórios demonstrativos por período/resíduo: quantidades produzidas, alternativas de gestão aplicadas, receitas ou custos acumulados.

Para o funcionamento adequado do sistema, possibilitando o cadastro cotidiano da geração dos resíduos e das saídas para destinação, um usuário administrador deve previamente cadastrar os resíduos que serão gerenciados, juntamente com seus detalhes, entre eles as classificações perante as normas vigentes e as alternativas de gestão para cada resíduo, incluindo seus detalhes entre eles as empresas terceirizadas envolvidas naquela destinação.

\section{Apresentação das telas e funcionalidades do sistema}

Segue na figura 3 os menus que disponibilizam o acesso às funcionalidades:

- Informações e Controle de Acessos ao sistema;

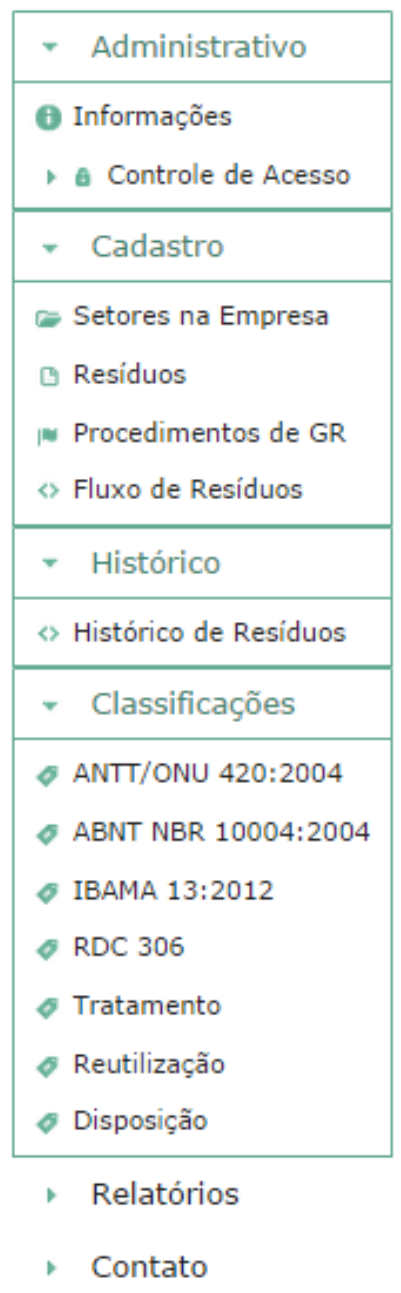

Figura 3: Menu de funcionalidades.
- Cadastro: Setores para organização e segregação da informação de geração;

- Cadastro dos resíduos e procedimentos de GR;

- Fluxo de resíduos: geração e destinação;

- Histórico: Acesso às informações de histórico de produção/destinação dos resíduos.

- Classificações: consulta a normas e leis vigentes relacionadas à gestão de resíduos.

- Relatórios: disponibilização e exportação de relatórios por resíduo e por período no formato "pdf".

Para o registro de produção devem ser informados, após a seleção do resíduo: volume, data e tipo de armazenamento.

$\mathrm{Na}$ figura 4 é apresentado a Tela de Cadastro de um novo resíduo: nome, composição, estado físico, se é orgânico, quais setores geram e as classificações. Na figura 4 é apresentado a Tela de Cadastro de um novo procedimento de gestão de resíduo, devem ser informados os campos: nome, descrição, se é do tipo Tratamento, Reutilização ou Disposição e qual o procedimento, quais as entidades envolvidas e seus papeis e permite também o armazenamento de arquivos. Nas figuras 5 e 6 estão representadas as Telas de Cadastro de fluxo de produção e destinação de resíduos.

Para o registro de destinação, devem ser informados, após a seleção do resíduo, os campos de volume, data e qual opção de gestão de resíduo, previamente cadastrada, foi efetivada.

Na figura 7 é apresentada a Tela de relatórios gráficos de um determinado resíduo para um determinado período mensal: linha do tempo, com navegação disponível, Gráfico diário e Gráfico acumulado.

\section{Apresentação de pontos fortes e fracos da ferramenta}

Alguns aspectos foram observados como pontos fortes, dentre eles a organização eficiente das informações, o cadastro de resíduos variados com alternativas distintas de gestão, a facilidade de acesso à informação, a presença de uma "inteligência central" que auxilia na tomada de decisões e a elaboração de relatórios que integra e apresenta os resultados.

Outras características da ferramenta podem ser configuradas como pontos fracos, dentre elas a não contemplação de todas as normas vigentes em relação a RSS, a não especificidade do Sistema para o gerenciamento de resíduos em saúde e/ou químicos e a ausência de funcionalidade para comparação de períodos de anos diferentes na mesma tela do relatório. 


\section{Cadastro Novo Residuo}
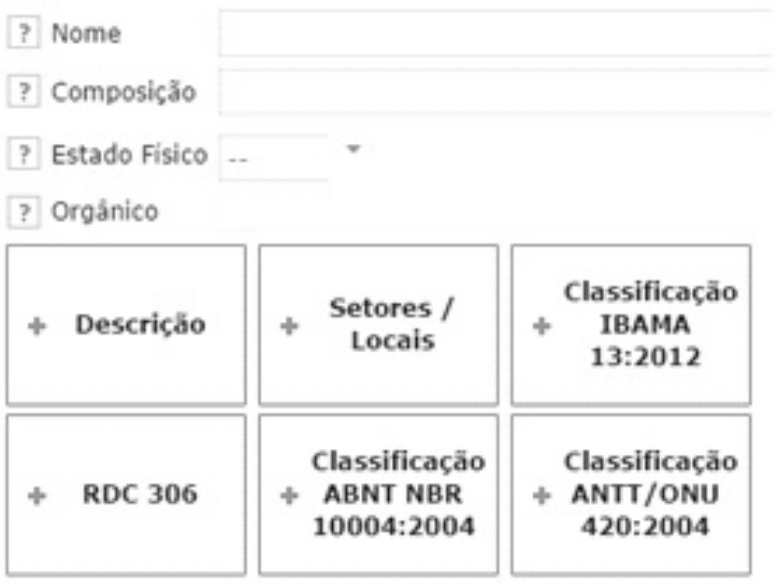

\section{Novo Procedimento de gestão de Residuo}

Residuo Selecionado: Residuos Perigosos de Medicamentos

Nome

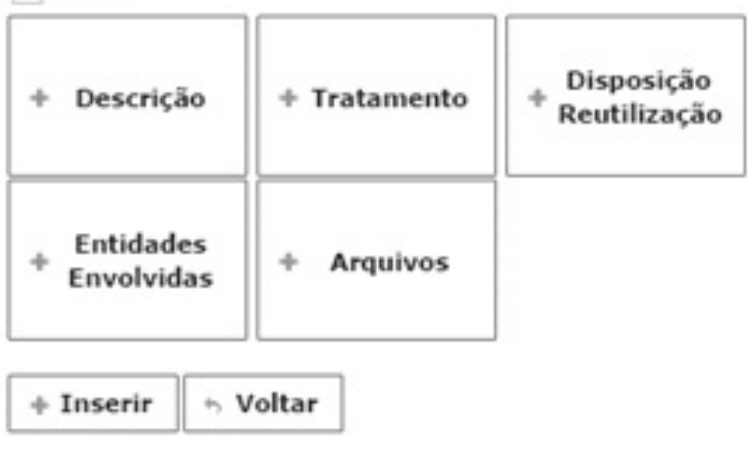

Figura 4: Telas para cadastro de resíduo e de procedimentos de GR.

registro de produçăo / saida de Residuo
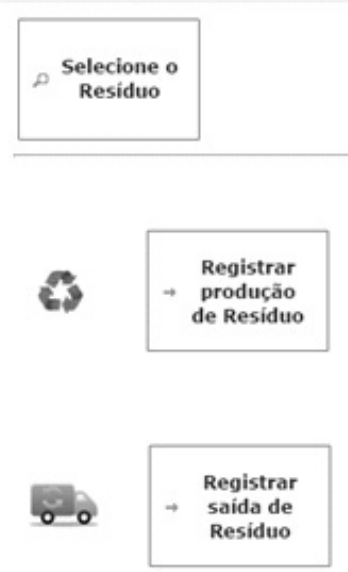

\section{Residuo Selecionado: \\ Residuos Perigosos de Medicamentos - RPM}

registros de produçáo de Residuos Perigosos de Medicamentos - RPM Data $\hat{v}$ Volume $c$

$\begin{array}{lllll}02 / 12 / 2013 & 7.1 \text { Quilograma (s) } & \Xi & \overline{\mathbf{x}} \\ 16 / 12 / 2013 & 5.8 \text { Quilograma (s) } & \overline{3} & \mathbf{\overline { x }}\end{array}$

registros de saida de Residuos Perigosos de Medicamentos - RPM Data $\odot$ Volume $\hat{\imath}$

Procedimento

31/12/2013 12.9 Quilograma (s) Tratamento medicamentos O Detalhes

Figura 4: Cadastro do fluxo de resíduos

Novo registro de produção de Residuo

Residuo: Residuos Perigosos de Medicamentos -

Volume $\quad 0,00$

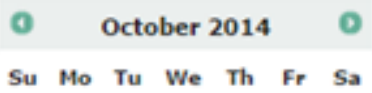

? Data

$\begin{array}{llllllll}5 & 6 & 7 & 8 & 9 & 10 & 11\end{array}$

$\begin{array}{lllllll}12 & 13 & 14 & 15 & 16 & 17 & 18\end{array}$

$\begin{array}{lllllll}19 & 20 & 21 & 22 & 23 & 24 & 25\end{array}$

$\begin{array}{llllll}26 & 27 & 28 & 29 & 30 & 31\end{array}$

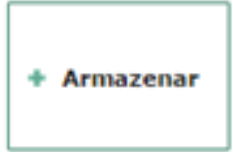

Novo registro de saida de Residuo

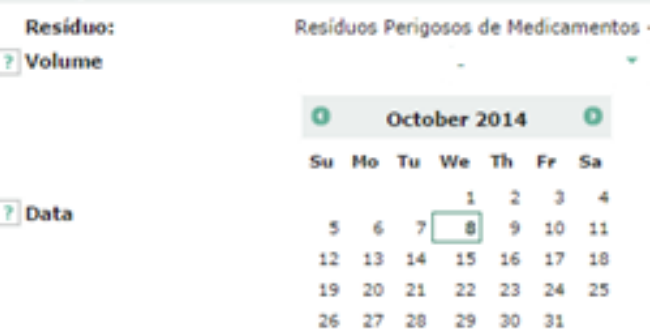

Receita (+) /Custo (-) RS

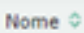

Selecione o Prc

Tratamento medicamentos Empresa Ambcamp vencedora dc

Figura 6: Cadastro de geração e destinação de resíduo. 


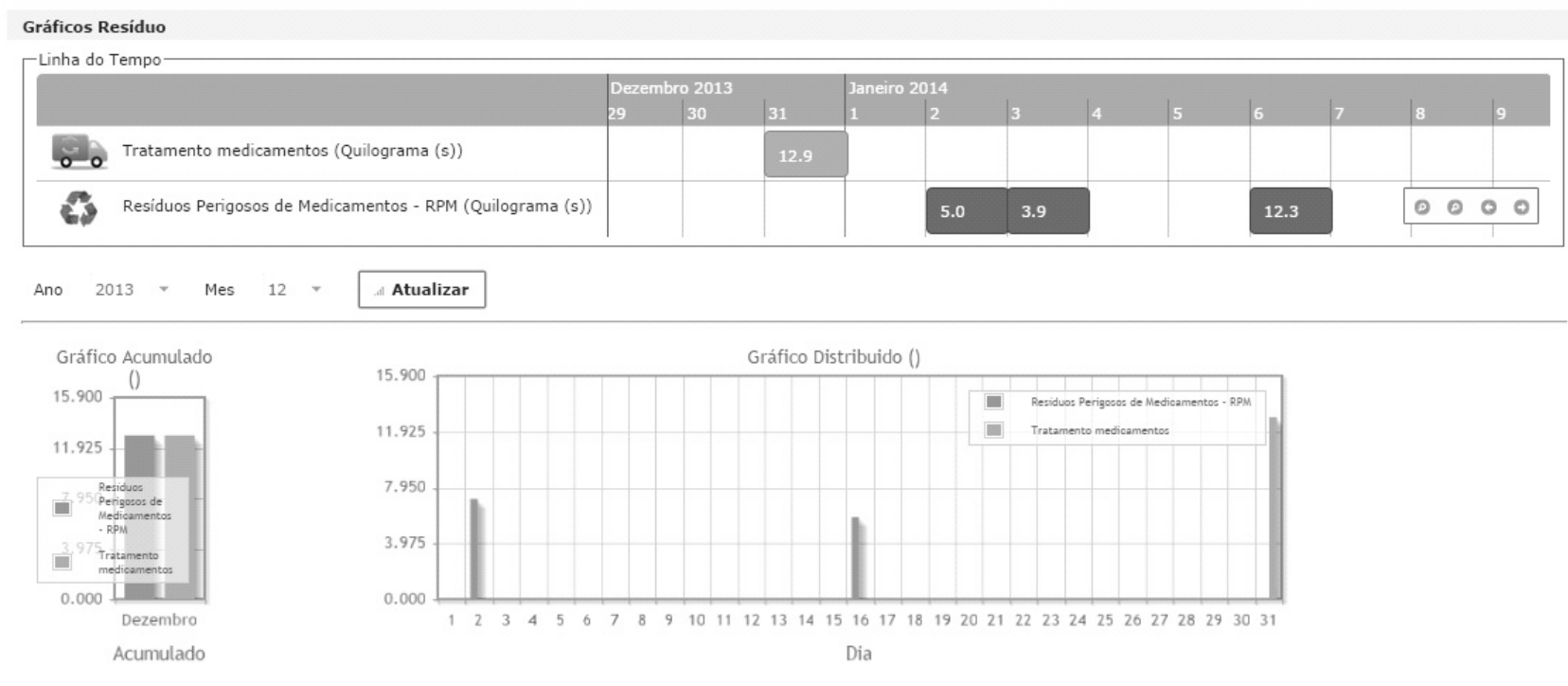

Figura 7: Tela de relatório de gráficos.

\section{Avaliação da ferramenta realizada pelo Ser- viço de GA}

O Serviço de GA considerou que a ferramenta: - É completa no sentido de que "traz informações sobre a legislação existente permitindo a gestão dos resíduos de maneira automatizada e estratificada entre os usuários e os tipos de resíduos produzidos";

- É funcional para o Serviço e de fácil utilização;

- Auxilia no poder de tomada de decisões, pois permite a rastreabilidade do processo;

- Apresenta telas que são pertinentes às fases dos processos envolvidos trazendo com clareza as informações;

- Permite rastreabilidade específica e efetiva, o que evita problemas de segregação e controle da Instituição;

- Reduz o consumo de papel.

Nesse contexto o Serviço de Gerenciamento Ambiental apresentou as seguintes sugestões:

- Acesso a telas específicas de acordo com a hierarquização do acesso às informações entre os usuários e a parte gerencial;

- Direcionamento para as legislações específicas para RSS, como a portaria 306/2004, CVS 21 e portaria 344/1998; ${ }^{14,15}$

- Disponibilização de informações cadastrais das empresas mais utilizadas no processo;

• Substituição do termo "produção" por "geração";
- Acréscimo de um campo que permita ao usuário o fornecimento de informações adicionais relevantes;

- Possibilidade de relatórios relacionados à geração, à área, período e acondicionamento dos resíduos;

- Relatórios informativos aos gestores referentes aos resíduos gerados e que possam requerer alguma intervenção;

- Relatórios que constem os usuários em comum para alguns produtos e para alguns resíduos específicos, como ao que se refere ao uso do formol;

Houve ênfase no aspecto inovador da ferramenta que permite a integração entre os usuários da rede no sentido de se manter atualizado e a troca de experiências.

\section{Discussão}

A questão ambiental vista como fundamental e o seu papel mais abrangente, despertou uma nova ótica do setor empresarial. A busca pelo desenvolvimento econômico e a preservação ambiental conduziram ao conceito de desenvolvimento sustentável. As adequações às legislações ambientais vigentes requerem investimentos. Os custos para tais finalidades tendem a aumentar na proporção que esses requisitos tornamse mais restritivos. Neste sentido, ações estratégicas que tratam a questão ambiental, especialmente os investimentos de maneira inovadora tornam possível obter redução de custos e reconhecimento público. A 
maneira como o Serviço de Gerenciamento Ambiental conduziu a sua cadeia produtiva, demonstrada na tabela 2 , permitem à área estar inserida no tripé da sustentabilidade ("triple bottomline") econômico, social e ambiental. ${ }^{16}$

Apesar de o Serviço ter sido premiado por suas ações inovadoras, observam-se alguns problemas, como a ausência de uma ferramenta administrativa específica que facilite a gestão de resíduos, como por exemplo, um sistema informatizado que poderia minimizar o déficit quantitativo de profissionais qualificados, uma vez que os existentes não prestam serviço em tempo integral, apenas como consultores técnicos.

A ferramenta sugerida vai de encontro às necessidades do Serviço, o que otimiza a execução das tarefas e agiliza os processos.

\section{Conclusão}

Um sistema informatizado com tais funcionalidades permite a gestão dos resíduos desde a identificação dos usuários e seus respectivos resíduos produzidos, da coleta até seu destino final possibilitando a rastreabilidade do processo, a tomada de decisão e ações corretivas e educativas quando pertinentes. Sugere-se a disponibilização do sistema para o Serviço de Gerenciamento Ambiental, por um período determinado, para uma avaliação mais profunda com apontamentos mais detalhados. Seria interessante também a realização de uma avaliação, juntamente com o setor de informática do HCFMRP, sobre o custo do desenvolvimento interno de uma ferramenta para os mesmos fins, versus o custo de compra e manutenção da ferramenta g2r pelo hospital.

\section{Referências}

1. Rodrigues POP. Uma abordagem sobre a gestão ambiental em serviços de saúde: Estudo nos laboratórios de análises clínicas, de anatomia patológica e citopatologia no município de Campos dos Goytacazes. In: Perspectivas on line. 2008; 5:(2). Disponível em: <http://www. seer. perspectivasonline. com. br/index. php/revista_antiga/article/view/306/219>. [Acesso em abr./2014].

2. São Paulo (Estado). Secretaria Do Estado De Meio Ambiente. ISO 14000: Sistema de Gestão Ambiental. In: Entendendo o Meio Ambiente. São Paulo. v. 14. 1998.

3. Esteves VA. Percepção do impacto de sistemas de gestão ambiental em hospitais. Dissertação (Mestrado) -Universidade Positivo, Curitiba, 2007.
4. Cesar RG. Diagnóstico ambiental interno visando a implantação futura de sistema de gestão ambiental no CETEM. In: Anais da XII Jornada de Iniciação Científica, jul 2004. CETEM, Centro de Tecnologia Mineral. Rio de Janeiro, 2004. p 1-10.

5. Faria HM, Silva RJ. Sistemas de gestão ambiental: Por que investir? In: Anais do Encontro Anual de Engenharia de Produção, 1998. Niterói, RJ 1998. Disponível em: http://www. abepro. org. br/biblioteca/ENEGEP1998_ART215. pdf [acessado em maio/2014].

6. Agencia Nacional de Vigilância Sanitária. ANVISA. Brasil. Resolução da Diretoria Colegiada $\mathrm{n} \cong 306$ de 07 de dezembro de 2004. Dispõe sobre o regulamento técnico para o gerenciamento de Resíduos de Serviços de Saúde. Diário Oficial da União 10 dez 2004. Seção 1. pg 49/56.

7. Ministério do Meio Ambiente. (Brasil). Conselho Nacional de Meio Ambiente. CONAMA. Resolução no 358 de 29 de abril de 2005. Dispõe sobre o tratamento final dos resíduos de serviços de saúde e dá outras providências. Diário oficial da União n 84 , 04 mai 2005, Seção 1. pg 63-65

8. ABNT - Associação Brasileira De Normas Técnicas. NBR 10004. Resíduos Sólidos: Classificação. Rio de Janeiro, $2^{\mathrm{a}}$ ed. 2004.

9. Agência Nacional de Vigilância Sanitária. ANVISA. Brasil. Manual de gerenciamento de resíduos de serviços de saúde. Brasília: Ministério da Saúde, 2006. Disponível em: <http:// www. anvisa. gov. br/servicosaude/manuais/manual_ gerenciamento_residuos. pdf>. [acessado em maio/2014].

10. Ventura, K. S. ; reis, L. F. R. ; Takayanagui, A. M. M. Avaliação do gerenciamento de resíduos de serviços de saúde por meio de indicadores de desempenho. In: Revista Engenharia Sanitária Ambiental Vol. 15 - $\mathrm{n}^{\circ} 2$ - abr/jun - Rio de Janeiro/RJ: ABES, 2010 pag. 167 - 176. Disponível: <http://www. scielo. br/pdf/esa/v15n2/a09v15n2. pdf> [Acesso abril/2014.

11. Brasil. Lei № 12. 305, de 02 de Agosto de 2010. Institui a Política Nacional de Resíduos Sólidos; altera a Lei № 9. 605, de 12 de fevereiro de 1998; e dá outras providências. Diário Oficial da União 03 ago 2010. Seção 1, pg 3.

12. Ministério do Meio Ambiente (Brasil). Conselho Nacional de Meio Ambiente. CONAMA. Resolução no 313 de 29 de outubro de 2002. Diário Oficial da União 22 nov. 2002, Seção 1, páginas 85-91.

13. Agência Nacional De Transportes Terrestres. ANTT. (Brasil). Resolução no 420, de 12 de fevereiro de 2004. Aprova as Instruções Complementares ao Regulamento do Transporte Terrestre de Produtos Perigosos. Diário Oficial da União no 103, 31 mai 2004. Seção 1, pg 84.

14. São Paulo (Estado). Portaria CVS-21, de 10 de setembro de 2008. Norma Técnica sobre Gerenciamento de Resíduos Perigosos de Medicamentos em Serviços de Saúde. Diário Oficial do Estado 11 ago 2008;p. 25.

15. Secretaria de Vigilância Sanitária (Brasil). Portaria n. 344 de 12 de maio de 1998. Aprova o Regulamento Técnico sobre Substâncias e Medicamentos Sujeitos a Controle Especial. Diário Oficial da União 19 mai 1998. Disponível em www. anvisa. gov. br [acessado em maio/2014].

16. Serviço Brasileiro de Apoio a Micro e Pequenas Empresas. SEBRAE. Brasil. Avaliação de Custos Ambientais. In: Gestão Ambiental Compromisso da Empresa, fascículo 6. 1996. 